
allemande

49-1 | 2017

Berlin 1957-1994

\title{
La politique culturelle à Berlin : du système de la RDA au système démocratique de l'Allemagne unifiée (1957-1994)
}

\section{Elisa Goudin}

\section{(2) OpenEdition}

\section{Journals}

Édition électronique

URL : https://journals.openedition.org/allemagne/519

DOI : 10.4000/allemagne.519

ISSN : 2605-7913

Éditeur

Société d'études allemandes

\section{Édition imprimée}

Date de publication : 16 juin 2017

Pagination : $37-55$

ISSN : 0035-0974

\section{Référence électronique}

Elisa Goudin, «La politique culturelle à Berlin : du système de la RDA au système démocratique de I'Allemagne unifiée (1957-1994) », Revue d'Allemagne et des pays de langue allemande [En ligne], 49-1 | 2017, mis en ligne le 16 juin 2018, consulté le 02 juin 2022. URL : http://journals.openedition.org/ allemagne/519; DOI : https://doi.org/10.4000/allemagne.519 


\section{La politique culturelle à Berlin: du système de la RDA au système démocratique de l'Allemagne unifiée (1957-1994)}

\section{- Elisa Goudin *}

La politique culturelle peut être considérée comme un des éléments centraux de la gouvernance urbaine, et ceci est d'autant plus vrai dans une ville comme Berlin, qui servait pendant la division de vitrine pour les deux États et est ensuite redevenue la capitale de l'Allemagne unifiée. Nous proposons donc de retracer ici les grandes lignes de cette politique ${ }^{(1)}$, avant et après la chute du Mur, afin de montrer comment ce secteur de l'action publique a été amené à se reconfigurer suite à la transition démocratique. Nous étudierons donc dans un premier temps les mesures de politique culturelle mises en place en RDA et leurs répercussions sur le cas particulier de Berlin-Est. Ce faisant, nous nous autoriserons ponctuellement des mises en perspective avec ce qui se passe de l'autre côté du Mur, car il est impossible de raconter une histoire de la politique culturelle est-allemande indépendante de celle de la RFA, qui présupposerait que les deux politiques sont des objets suffisamment étanches l'un à l'autre pour être étudiés dans une perspective uniquement comparatiste ou en termes de transferts ${ }^{(2)}$. Dans un second temps nous examinerons les mesures de transition mises en place au moment de la réunification afin de redéfinir des modalités d'action de l'État sur la culture qui puissent

* Maître de conférences en études germaniques, Université Sorbonne Nouvelle-Paris 3.

1 Les trois tomes de Dokumente zur Kunst-, Literatur-, und Kulturpolitik der SED constituent vraisemblablement la source la plus exhaustive de documents officiels sur cette question: Elimar Schubbe (dir.), t. 1 : 1946-1970, Stuttgart, Seewald, 1972; Gisela Rüss (dir.), t. 2 : 1971-1974, Stuttgart, Seewald, 1976; Peter Lüвве (dir.), t. 3: 1975-1980, Stuttgart, Seewald, 1984.

2 Nous proposons donc de nous inspirer de la méthode dite de «l'histoire croisée» développée par M. Werner et B. Zimmermann à l'EHESS et qui vise à adopter des perspectives multiples afin de dépasser l'écriture nationale de l'histoire. En effet, l'une des limites de l'approche comparée entre histoires nationales ou de l'histoire des transferts culturels est qu'elles risquent de présupposer que leurs objets d'études sont a priori distincts et peuvent être saisis en termes de transferts alors que l'on sait que les histoires nationales, surtout pour la RDA et la RFA, étaient très imbriquées, en particulier lorsqu'on s'intéresse à la ville de Berlin. 
éviter que ne se perde la "substance culturelle est-allemande», conformément à ce qui est stipulé à l'article 35 du traité d'unification entré en vigueur le 3 octobre 1990.

\section{La politique culturelle dans Berlin divisé (1957-1990)}

Ce n'est qu'en 1965 qu'a lieu le $11^{\mathrm{e}}$ plenum du comité central du SED (Kahlschlagplenum), qui durcit l'appareil de répression pour infiltrer et neutraliser toute forme d'opposition, notamment dans le domaine artistique, mais déjà dans les années 1950 les fondamentaux de la politique culturelle de la RDA étaient en place. Cette politique culturelle avait une fonction idéologique, elle constituait un instrument de propagation de l'orthodoxie politique: promouvoir «la culture socialiste», perçue comme un socle sur lequel s'enracine le projet plus global de développement d'une société socialiste.

Un autre pilier de cette politique culturelle est l'idée de favoriser un échange fructueux entre l'art professionnel et l'art populaire, «Berufskunst» et «Volkskunst». Il s'agit de créer un quotidien socialiste où l'art a une place importante, et ceci reste une constante jusqu'à la fin. Un ouvrage collectif publié en 1989 par «l'Institut pour la recherche sur l'art populaire» de $\mathrm{RDA}^{(3)}$ cherche à définir une stratégie de développement de l'art populaire pour les années 1990, en s'appuyant sur les acquis de la quatrième conférence sur l'art populaire, IV. Volkskunstkonferenz. La notion de création populaire, kulturelles Volksschaffen, y est présentée comme un outil de démocratisation sociale, dans la mesure où l'art devient accessible à tous les citoyens. À Berlin-Est, on a ainsi créé la Berliner Haus für Kulturarbeit, Maison berlinoise du travail culturel, fondée en $1953^{(4)}$ sous le nom de Berliner Volkskunstkabinett et dissoute en 1991. L'objectif était de fédérer les différents collectifs artistiques de la ville, d'organiser tous les ans un concours du meilleur cercle d'ouvriers écrivains, peintres, etc. La Maison berlinoise du travail culturel s'occupait aussi de faire vivre des festivals, des fêtes de l'art populaire, préparées dans ces collectifs artistiques par les ouvriers et des artistes de métier qui devaient travailler ensemble ${ }^{(5)}$. Par ailleurs elle a aussi été responsable de la politique de la mémoire, en organisant par exemple les festivités pour les 750 ans de la ville de Berlin-Est, dans un esprit de surenchère face à ce qui se passait à Berlin-Ouest. À Berlin, ville-vitrine pour les deux États, la politique culturelle est constamment liée au contexte de rivalité entre les deux États allemands sur le plan de l'acquisition d'une légitimité internationale. La RDA cherchait à saboter la prétention de la RFA à représenter seule la nation allemande à un niveau international. La RFA a en effet toujours prétendu être le seul État héritier de l'Allemagne antérieure au Troisième Reich et s'est d'emblée constituée comme le prolongement unique de la nation allemande et donc des différentes incarnations de cette nation dans des États au gré des évolutions historiques ${ }^{(6)}$.

3 Cf. Beiträge zur Strategie des künstlerischen Volksschaffens für die neunziger Jahre, Wissenschaftliche Beiträge, Heft 19, Institut für Volkskunstforschung beim Zentralhaus für Kulturarbeit der DDR, Leipzig, Zentralhaus-Publikation, 1989.

4 Elle a été créée en janvier 1953, donc avant le contexte traumatique généré par le soulèvement de juin et sa répression sanglante.

5 La lecture des archives de cette institution montre que cette coopération n'a pas toujours été facile.

6 C'est ce que l'on nomme en allemand «Alleinvertretungsanspruch», la prétention de la RFA à représenter seule l'Allemagne dans son ensemble. 
Cette conception de la culture comme instrument de propagation de l'orthodoxie politique implique une approche normative de l'art, qui repose sur une dichotomie entre le politiquement souhaitable et le politiquement nuisible dans l'art. Des rapprochements ont même parfois été établis sur ce point avec l'approche normative de l'art sous le régime national-socialiste qui prétendait définir un art «sain » et un art «malade», nommé "art dégénéré» (entartete Kunst). Indépendamment de la question de savoir si ce rapprochement est légitime ou non, il importe de comprendre les enjeux de cette fonction idéologique de la politique culturelle en RDA: l'art devait acquérir un rôle d'éducateur, il devait permettre d'ancrer la "morale socialiste» dans les consciences, de transformer cette morale en une expérience quotidienne, et donc d'éviter que les tentations de remise en cause de cette idéologie ne se transforment en une véritable opposition politique. Tout se passe comme si le Parti partait systématiquement du postulat que la population était a priori très réceptive aux idées qu'il combat ${ }^{(7)}$. L'existence de la RDA, qui n'a jamais été légitimée par les urnes, n’était garantie que par la seule puissance militaire de l'URSS. Les dirigeants est-allemands étaient parfaitement conscients du manque d'adhésion populaire.

Il faut noter que cette volonté du Parti d'imposer sa domination sur la culture est apparue très tôt: dès l'été 1945, le poète communiste Johannes R. Becher crée la Confédération culturelle pour le renouveau démocratique de l'Allemagne ${ }^{(8)}$ (Kulturbund zur demokratischen Erneuerung Deutschlands). Son ancêtre est l'Aide internationale des travailleurs, Internationale Arbeiterhilfe, fondée en 1921 par Willy Münzenberg, dans l'objectif de diffuser des livres, films, et revues diverses à la classe ouvrière. Ensuite, en juillet 1946, le Parti socialiste unifié, SED (sozialistische Einheitspartei), organise une commission culturelle (Kulturausschuss). Progressivement, toutes les organisations de masse du SED vont créer et entretenir leurs propres commissions culturelles ${ }^{(9)}$. C'est ainsi que furent créées au début des années 1950 une "Commission étatique pour les affaires artistiques» (Staatliche Kommission für Kunstangelegenheiten) et une "Administration pour la littérature et l'édition» (Amt für Literatur und Verlagswesen), de même qu'une Académie des sciences fondée en 1946 et une Académie des arts en 1950. Enfin, le SED créa en 1954 le ministère de la Culture, qui se substitua à la Commission étatique pour les affaires artistiques ${ }^{(10)}$.

La question essentielle qui se pose alors pour l'analyse de la politique culturelle du SED est celle de la censure. En juin 1971, Erich Honecker déclare qu'il ne doit plus y avoir de tabous dans le domaine culturel, il encourage les artistes à s'exprimer librement

7 Cf. Jacques Poumet, «Sous l'œil de la censure: la série de courts métrages Das Stacheltier», Allemagne d'aujourd'hui, nº 128 (1994), p. 120-130.

8 Pour de plus amples détails sur cette institution, on pourra se reporter au manifeste qui a été publié lors de sa fondation, en 1945: Manifest des Kulturbundes zur demokratischen Erneuerung Deutschlands, Berlin-Est, Aufbau, 1945.

9 Cf. Manfred JäGEr, Kultur und Politik in der DDR. Ein historischer Abriss, Cologne, Wissenschaft und Politik, 1982.

10 Le soulèvement en Hongrie au cours de l'automne 1956 explique également le durcissement de la politique culturelle du SED. On peut lire des symboles de ce durcissement dans l'arrestation de deux personnages importants de la vie littéraire est-allemande: le philosophe Wolfgang Harich et le directeur de la maison d'édition Aufbau, Walter Janka. 
et même à présenter une «vision critique de la société socialiste et de ses contradictions " ${ }^{(11)}$. Pourtant, si l'on analyse les faits, aucune libéralisation ne s'est produite suite à cette déclaration d'intention. Le remplacement en 1971 d'Ulbricht par Honecker à la tête de l'État et du Parti n'a pas apporté le dégel dans le domaine artistique auquel certains s'attendaient. On peut au contraire parler d'un durcissement supplémentaire. Si la censure proprement dite n'existait officiellement pas en RDA, les écrivains étaient néanmoins contraints de présenter leurs manuscrits à la Direction de l'édition et de la distribution du livre, Hauptverwaltung Verlage und Buchhandel, qui pouvait refuser ou accorder l'autorisation officielle de publication, Druckgenehmigung. Il existait donc à l'évidence une censure de facto. De même, les galeries d'art par exemple étaient soit des galeries dépendant de l'Organisation nationale du commerce de l'art de RDA, staatlicher Kunsthandel der DDR, soit des galeries communales, gérées par les municipalités, toujours sous le contrôle strict des autorités de l'État, comme le Studio Bildende Kunst à Berlin-Est. Il existait par ailleurs de très rares galeries privées en dehors de Berlin telles que celle de Judy Lybke à Leipzig, la galerie Kuehl à Dresde, ou la galerie Klara Mosch ${ }^{(12)}$ à Karl Marx-Stadt/Chemnitz, mais ce sont vraiment des exceptions. Le Studio Bildende Kunst de Berlin-Est était nettement plus représentatif de la réalité du quotidien des galeries d'art en RDA, où la mainmise de l'État était constante.

La politique culturelle de l'État a progressivement été transférée au ministère de la Sécurité d'État, Ministerium für Staatssicherheit (Stasi), et elle s'est ainsi dotée d'une dimension nouvelle, celle d'une force de surveillance, de conspiration. Les collaborateurs officieux de ce ministère, Inoffizielle Mitarbeiter (IM), ont constamment cherché à infiltrer les milieux artistiques, en particulier la scène littéraire, en utilisant le chantage et l'espionnage. De nombreux artistes et intellectuels critiques vis-à-vis du régime vivaient à Berlin-Est, c'est la raison pour laquelle la Stasi était particulièrement mobilisée dans les institutions culturelles berlinoises, même si des phénomènes comparables étaient à l'œuvre sur tout le territoire de la RDA. Walther Joachim a montré, dans l'ouvrage intitulé Sicherungsbereich Literatur. Schriftsteller und Staatssicherheit in der DDR ${ }^{(13)}$, la façon dont le ministère de la Sécurité d'État concevait son rôle à l'égard des écrivains, et notamment la façon dont la police secrète tentait d'établir qui pensait quoi, et, parfois, mettait en scène des procès politiques qui, à 43 reprises, ont abouti entre 1945 et 1989 à ce que des écrivains soient condamnés à des peines de prison. Grâce à ce travail, on sait comment fonctionnait la sous-division de la Stasi responsable de la surveillance des écrivains, et qui s'appelait Hauptabteilung XX (depuis $1964 \mathrm{HA} \mathrm{XX/7).} \mathrm{À} \mathrm{l'aide} \mathrm{des}$ archives des cadres employés dans cette section, W. Joachim reconstitue dans son chapitre «Der Apparat» l'origine et la mentalité de ces cadres et montre l'ampleur de leur désintérêt pour les questions littéraires et même culturelles en général, toutes générations confondues ${ }^{(14)}$, et leur peur que des facteurs de déstabilisation venus de Berlin-Est

11 Cf. G. Rüss, Dokumente (note 1), p. 287.

12 Ce nom fait allusion à une figure de l'histoire et est composé des premières lettres des noms des artistes: Cla(us), Ra(nft), Mo(rgner), Sch(ade).

13 Walther JoАснім, Sicherungsbereich Literatur. Schriftsteller und Staatssicherheit in der DDR, Berlin, Ch. Links Verlag, 1996.

14 Il distingue les «Pioniere», qui ont, dans les premières années de la RDA, mis en place ce système de persécution des écrivains non conformes à la ligne du Parti, puis les «Profis» entre 1969 et 1978 et enfin le «Nachwuchs» qui a travaillé dans les années 1980 dans cette section. 
ou d'autres grandes villes ne contaminent le reste de la RDA. Mais cette étude se base uniquement sur les archives de la Stasi et ne prétend donc pas analyser comment les directives de la Stasi ont été reçues par ces agents, ni si et à quelles conditions des marges de manœuvre existaient pour contourner ces directives. Or, les archives de la Berliner Haus für Kulturarbeit montrent que des marges de manœuvre existaient ${ }^{(15)}$.

$\mathrm{Au}$ total, la culture est donc appréciée à Berlin-Est et plus généralement en RDA uniquement pour son "efficacité» sociale, c'est-à-dire pour ses répercussions sur l'adhésion du peuple aux principes posés par le régime. Le poème de Bertolt Brecht intitulé «La solution » est à cet égard très révélateur. Bertolt Brecht choisit de dénoncer, sur le mode de l'ironie, les apories de l'approche de l'éducation populaire choisie par le régime: utiliser la politique culturelle comme vecteur d'une «morale» revient finalement à nier toute autonomie au peuple lui-même, ce que Bertolt Brecht radicalise en proposant que le Parti choisisse un autre peuple sur lequel ses stratégies d'éducation fonctionneraient mieux.

L'ensemble des critères appliqués à l'art a été résumé sous le terme de réalisme socialiste, qui se caractérise d'abord par le principe de l'optimisme, de l'exemplarité, du héros positif au théâtre par exemple. Le regard de l'artiste devait constamment être "tourné vers un avenir radieux» ${ }^{(16)}$, rendu possible par le passage au socialisme. On demandait aussi à l'artiste de ne pas s'attacher à l'expression des exigences individuelles mais seulement à celle des besoins collectifs. Cette exigence est également formulée à plusieurs reprises dans les rapports établis à la suite des conférences sur l'art populaire de $\mathrm{RDA}^{(17)}$. En voici l'une des occurrences les plus significatives:

«Nous, les participants de la quatrième conférence sur l'art populaire, nous nous adressons à tous ceux qui produisent de l'art populaire en RDA: [...] Nous voulons rendre tangibles par le biais de notre activité artistique les avantages du socialisme et la beauté de notre patrie, nous voulons aider à faire découvrir les trésors de notre patrimoine culturel humaniste, propager l'optimisme, la joie de vivre et l'assurance de la victoire, approfondir la coopération étroite et fraternelle et l'amitié à toute épreuve qui nous lient à l'Union soviétique et aux autres pays de la communauté d'États socialistes ${ }^{(18)}$.

15 Cf. Elisa Goudin-Steinmann, «Gedächtnis und Aneignung der Geschichte in einer kulturellen Organisation der DDR in den 1980er Jahren: das Berliner Haus für Kulturarbeit », in: Oliver Dimbath, Hanna HaAg, Nina Leonhard, Gerd Sebald (dir.), Organisation und Gedächtnis. Über die Vergangenheit der Organisation und die Organisation der Vergangenheit, Stuttgart, Springer VS, 2016, p. 225-248; Elisa Goudin-STEINMANN, "Subversion, détournement et opposition: l'exemple de quelques Kulturhäuser de Berlin-Est dans les années 1980 », in : Hélène CAmarade et Sybille GoepPER (dir.), Résistance, dissidence et opposition en RDA, 1949-1990, Villeneuve d'Ascq, Presses universitaires du Septentrion, 2015, p. 199-212.

16 Cette expression est récurrente dans les écrits émanant des autorités proches du SED, on pourra se reporter en particulier à l'ouvrage suivant: Dokumente zur Kulturpolitik der SED, 1971-1986, Zentralrat der FDJ, Berlin-Est, Dietz, 1986.

17 Cf. par exemple: Dokumente und Materialen der IV. Volkskunstkonferenz der DDR, Gera, $1^{\text {er }}$ décembre 1984, Wissenschaftliche Beiträge, Heft 14, Leipzig, Zentralhaus-Publikation, 1985.

18 «Wir Teilnehmer der IV. Volkskunstkonferenz wenden uns an alle Volkskunstschaffenden der DDR: [...] Wir wollen mit unserem künstlerischen Schaffen die Vorzüge des Sozialismus und die Schönheit unserer Heimat erlebbar machen, die Schätze unseres humanistischen Kulturerbes entdecken helfen, Optimismus, Lebensfreunde und Siegeszuversicht verbreiten, die enge, brüderliche Zusammenarbeit 
Cette approche de l'art condamnait aussi d'emblée toutes les tendances non figuratives. Ainsi, lors du troisième congrès du Parti en 1952, le SED a condamné en bloc ce qu'il nommait la «décadence de l'image humaine», «les théories du cosmopolitisme hostiles au peuple», «l'objectivisme bourgeois» et «la préférence pour le laid et l'absurde», pour ne citer que les expressions les plus représentatives de l'argumentation développée ${ }^{(19)}$. Le refus de toute prise de distance face à la réalité immédiate se lit aussi dans la véhémence des attaques contre le théâtre de Bertolt Brecht, et en particulier contre son concept de «distanciation» (Verfremdungseffekt), qui avait justement pour objectif de briser la perspective aristotélicienne du théâtre comme imitation de la réalité( ${ }^{(20)}$.

À cet égard, Berlin-Est a constitué un point central de ces débats sur le formalisme, car c'est à Berlin-Est qu'était située la Hochschule für angewandte Kunst BerlinWeißensee, dont un peintre comme Horst Strempel, parti vivre en 1947 dans ce qui deviendra la RDA, deviendra un professeur d'arts plastiques influent avant de quitter la RDA en 1953 en raison de divergences fondamentales sur le refus du formalisme dans l'art, qu'il considérait comme une absurdité.

L'ensemble de ces exigences à l'encontre de la création artistique constitue ce qu'il est courant de nommer le Bitterfelder Weg. Cette approche normative de tout processus de création artistique trouve peut-être sa source dans une lettre célèbre publiée en 1955 par une bibliothécaire de la ville de Nachterstedt, en Saxe-Anhalt, dans la revue Tribüne ${ }^{(21)}$. Dans cette lettre ouverte, elle reproche aux écrivains de RDA de ne pas avoir su abandonner «le goût bourgeois» et se «conformer à la base», c'est-à-dire se

und unverbrüchliche Freundschaft mit der Sowjetunion und den anderen Ländern der sozialistischen Staatengemeinschaft vertiefen », ibid., p. 5.

19 Dokumente der SED, vol. III, Berlin-Est, 1952, p. 118 sq.

20 Brecht a été invité dès la fin de la guerre à rentrer en RDA pour contribuer à la construction du pays et mettre en scène lui-même ses pièces de théâtre. Il a accepté et en octobre 1948, à l'invitation du Kulturbund zur demokratischen Erneuerung Deutschlands, qui se nommera ensuite Kulturbund der $D D R$, il rentre de Zurich à Berlin en passant par Prague. Son nom est dès lors associé au Berliner Ensemble sur le Schiffbauerdamm. Il y développe sa théorie du théâtre épique et met notamment en scène, avec Erich Engel, Mutter Courage und ihre Kinder, dont la première a lieu dès janvier 1949 et obtient un succès immédiat et considérable. Pourtant, les premières esquisses des futurs conflits avec les fonctionnaires de la culture de RDA se font déjà jour: on commence à lire dans les archives des concepts comme celui de "volksfremde Dekadenz», etc. Mais Brecht continue le travail et s'entoure d'élèves comme Benno Besson, Peter Palitsch et Egon Monk. Il travaille aussi avec Jakob Lenz sur la mise en scène de la pièce Der Hofmeister. Pendant toutes ces années, et malgré le durcissement des principes du SED en matière de politique culturelle, Brecht réussit à poursuivre dans la voie qu'il s'est tracée, par une politique des petits pas, qui lui permet de ne pas trop subir les reproches de formalisme que d'autres auteurs comme Paul Dessau vont subir. Il a toujours agi de façon très habile, cherchant le compromis, même après que le SED a cherché, en distribuant des cartons d'invitation à des personnes mal intentionnées, à organiser un échec lors de la première de la pièce Das Verhör des Lukullus le 17 mars 1951. Cette attitude lui permit même d'obtenir le Nationalpreis der DDR I. Klasse fin 1951. Cela n'a pas été le cas pour tous les auteurs de ce que l'on pourrait appeler l'avant-garde artistique de RDA. L'ouvrage d'Alfred Kurella, qui était responsable de la culture au SED et membre du Bureau politique dans les années 1960, montre que des auteurs comme Ionesco et Beckett ou un peintre comme Miro étaient considérés comme «des produits extrêmes de la décadence», cf. Alfred Kurella, «Zum Problem der Dekadenz», Einheit, 5 (1958), p. 749.

21 «Offener Brief an unsere Schriftsteller», Nachterstedter Brief, 27.01.1955, in: E. Schuвве, Dokumente (note 1), t. 1, p. 350 . 
tourner de façon définitive vers le peuple lui-même. Cette lettre ouverte est devenue l'un des symboles du Bitterfelder Weg, dont elle est probablement, au moins en partie, à l'origine ${ }^{(22)}$. Le topos de la mise en conformité de l'écriture littéraire et de «la base» y est déjà développé.

Le corollaire de cela est la volonté de permettre par la politique culturelle l'avènement d'un citoyen nouveau, à la fois travailleur manuel et artiste. On retrouve cette idée dans le terme Volkskunst: un art que les ouvriers se seraient approprié, qui ne serait plus l'apanage d'une élite. Le mot d'ordre formulé pour la première fois lors de la conférence de Bitterfeld en 1959, "Greif zur Feder, Kumpel!", "À vos plumes, camarades!», résume à lui seul l'utopie de l'avènement d'une culture nouvelle. Le symbole le plus marquant de cette orientation est le mouvement dit des ouvriers écrivains (schreibende Arbeiter), qui se développe dans le sillage de cette conférence. Les ouvriers étaient incités à écrire, avec l'idée sous-jacente que cette littérature serait plus conforme à l'idéal d'expression du «socialisme réellement existant». C'est ce principe qui définissait le concept d'«agit-prop» en Union soviétique, fondé sur une volonté de diffusion des idées révolutionnaires sur les lieux de travail ${ }^{(23)}$. En RDA, chaque entreprise d'envergure devient ainsi le lieu d'un développement de la vie culturelle du quartier où elle est implantée, grâce aux bibliothèques et aux maisons de la culture qui constituent de nouveaux centres culturels et se développent dans le cadre de ces entreprises (VEB).

Dans un article intitulé «La RDA, un Kulturstaat ouvrier? », Sandrine Kott souligne l'envergure de ce phénomène:

«En mars 1957, lors d'une conférence sur l'art populaire organisée par le syndicat, Rentzsch, alors responsable de la culture, recense 4500 chœurs, 3000 troupes de danse et 1500 troupes dramatiques. Quelque 40000 personnes seraient alors impliquées dans l'élaboration d'une nouvelle culture socialiste»" ${ }^{(24)}$.

Il y a plusieurs exemples concrets à Berlin-Est des modalités de ce transfert de l'activité artistique vers les entreprises. S. Kott s'appuie notamment sur l'entreprise Bergmann Borsig de Berlin qui accueille en 1950 la troupe du Deutsches Theater sous la direction de Wolfgang Langhoff ${ }^{(25)}$. Les personnes concernées sont souvent devenues des experts dans le maniement du double-sens, de l'ironie. Elles recherchaient souvent dans ces collectifs artistiques le second degré, la parodie. Cette «culture du contre» profondément subversive est surtout le fait de ceux qui sont nés sous la RDA et n'ont donc connu que cette référence politique, que l'on nomme les Hineingeborene. C'est ainsi que le quartier de Prenzlauer Berg à Berlin-Est se transforme en une véritable métaphore, qui symbolise une non-légalité en dehors de la culture officielle cautionnée par l'État. Une rétrospective a été consacrée à cette culture parallèle en 1997 au musée historique allemand de Berlin sous le titre «Bohème et dictature en RDA».

22 Pour de plus amples détails, on pourra se reporter à l'article de Jean Mortier, «La lettre de Nachterstedt, coup d'envoi du Bitterfelder Weg», Allemagne d'aujourd'hui, n 129 (1994), p. 126-141.

23 Cette conception était en particulier celle du chef de la commission culturelle du Bureau politique du SED, Alfred Kurella.

24 Sandrine Kотт, «La RDA, un Kulturstaat ouvrier?», Documents, 1 (1999), p. 51.

25 Ibid., p. 50. 
Matthias Theodor Vogt évoque les conséquences en termes de pratiques sociales:

«L'objectif du travail culturel de masse, organisé de part en part, était de constituer un substitut à ce que le système refusait: la rencontre du citoyen avec lui-même au sein d'une communauté autonome. L’effet secondaire a été une culture des niches très diversifiée ${ }^{(26)}$.

Au total, ce qui caractérisait la politique culturelle à Berlin-Est, c'est qu'elle dépassait largement le cadre strict d'une politique culturelle "traditionnelle» et englobait tous les aspects de la vie sociale.

Sur le thème de l'unité culturelle nationale, la position de la RDA n'a pas été constante. Si, jusque dans les années 1950, on chantait encore l'hymne national contenant l'expression «Deutschland, einig Vaterland» et si l'on revendiquait encore une unité nationale basée sur le socialisme, cela n'est plus du tout le cas ensuite. Seule la musique de l'hymne écrit par Johannes R. Becher est encore autorisée et il n'est plus question de se référer à une unité culturelle entre les deux États. L'ouvrage de E. Hexelschneider et E. John intitulé La culture comme lien? Une analyse de la thèse de la "nation culturelle allemande unitaire» ${ }^{(27)}$ illustre bien ce refus de la thèse de la nation culturelle allemande qui aurait persisté malgré la division du pays en deux États, thèse qui est celle qui caractérise la position officielle de la RFA.

«De même qu'une nation socialiste, dont les caractéristiques fondamentales ont été et sont toujours déterminées par la classe ouvrière victorieuse, se développe en RDA, une culture nationale allemande socialiste a également vu le jour» ${ }^{(28)}$.

Les auteurs font en particulier référence à un article du Frankfurter Rundschau du $1^{\text {er }}$ décembre 1978 qui explique les motivations qui ont conduit à la signature par les ministres de la Culture ${ }^{(29)}$ des Länder d'un texte sur la façon dont la «question allemande» devait être traitée en cours, en insistant en particulier sur les notions de «nation culturelle» et de «culture nationale allemande unitaire». E. Hexelschneider et E. John ne nient pas qu'il puisse exister des liens affectifs individuels qui ont survécu à la division de l'Allemagne, mais ils ajoutent aussitôt: «Mais nous refusons catégoriquement d'utiliser ces sentiments au service d'objectifs revanchistes et de les estampiller du sceau d'une appartenance nationale commune» ${ }^{(30)}$. L'idée de l'existence d'une unité culturelle est analysée comme un point de vue bourgeois: la classe bourgeoise a utilisé à son profit les sentiments d'appartenance nationale dans le passé, et ceci à plusieurs

26 «Ziel der durchorganisierten Breitenkulturarbeit war ein Ersatz für die vom System nicht erwünschte Selbstfindung der Bürger in selbstorganisierter Gemeinschaft, Nebenergebnis war eine vielfältige Nischenkultur», in: Matthias Theodor Vogt (éd.), Kulturräume in Sachsen. Eine Dokumentation zur Genese des sächsischen Kulturraumgesetzes und zum «Probejahr» 1995, Universitätsverlag Leipzig, 1996 , p. 25.

27 Erhard Hexelschneider et Erhard John, Kultur als einigendes Band? Eine Auseinandersetzung mit der These von der "einheitlichen deutschen Kulturnation», Berlin, Dietz Verlag, 1984.

28 «In dem Maße [...], wie sich in der DDR die sozialistische Nation entwickelt, deren Wesenszüge von der siegreichen Arbeiterklasse bestimmt wurden und werden, bildete sich auch eine sozialistische deutsche Nationalkultur heraus", ibid., p. 5.

29 Il s'agit en fait des «Kultusminister».

30 «Aber wir wenden uns dagegen, solche Gefühle [...] für revanchistische Zwecke auszunutzen und sie mit dem Stempel nationaler Gemeinsamkeiten zu versehen », in: Hexelschneider/John, Kultur als einigendes Band? (note 27), p. 6. 
reprises, et c'est exactement le même processus qui est en train de se répéter. La classe bourgeoise se réfère de façon artificielle à une prétendue unité culturelle afin de se légitimer elle-même. Ce faisant, elle refuse de reconnaître le processus objectif de formation d'une culture socialiste nationale en RDA afin de servir ses propres fins.

La RDA s'efforce en fait de démasquer derrière l'idéal d'échanges culturels et derrière la thèse ouest-allemande de la nation culturelle unitaire une nouvelle variante de la stratégie globale d'agression de la RFA, et du monde impérialiste tout entier contre la RDA :

«Par le biais du concept ethnique de "l’Allemand" existant par-delà les frontières étatiques, on fait revivre, voire on entretient ("Mais nous sommes bien tous des Allemands") des émotions, qui sont censées suggérer un sentiment d'appartenance commune à l'Est et à l'Ouest. Ceci est lié à un point de vue revanchiste, que caractérise particulièrement bien la formule "l'Allemagne, l'Allemagne par-dessus tout" " ${ }^{(31)}$.

Pour résumer, le point de vue de la RDA sur la question de l'unité culturelle allemande consiste essentiellement à affirmer que la RFA développe une idéologie de l'Allemagne, qui est dépassée historiquement mais maintenue juridiquement et politiquement par la classe dirigeante bourgeoise. L'apparition d'une culture nationale en RDA est donc aux yeux du SED à la fois une conséquence et une cause de la transformation sociale, de la prise de conscience par les travailleurs de leur «rôle historique». C'est la raison pour laquelle le SED insiste sur le fait qu'il n'y a jamais eu dans l'histoire allemande de nationalisme bourgeois au contenu démocratique, car dès le début, celui-ci est allé de pair avec une «arrogance nationaliste» et une "définition romantique de la germanité» ${ }^{(32)}$. Par ailleurs, les structures socio-économiques des deux États allemands étant désormais antagonistes, l'idée même d'unité culturelle est un non-sens du point de vue du SED:

«Il ne peut y avoir d'unité culturelle [...] et idéologique entre des classes antagonistes qui vivent ensemble dans une même nation, et donc encore moins entre des nations aux organisations sociales différentes. Dans les sociétés de classes, la culture a toujours un caractère de classe ${ }^{(33)}$.

Cependant, la notion de Kulturnation n'a pas cessé d'être une notion problématique en RDA, notamment après la signature du traité interallemand en 1972. Il n'était pas possible de nier totalement l'existence d'un Panthéon littéraire commun, ni d'empêcher que ne circule le discours de la RFA autour de l'Allemagne comme nation culturelle, ne serait-ce que par des médias comme la télévision. Suite à ce traité, le thème

31 «Mit dem ethnischen Begriff des über Staatsgrenzen hinweg existierenden 'Deutschen' werden Emotionen wachgerufen oder sogar bewahrt ('Wir sind doch alle Deutsche'), die ein nationales Zusammengehörigkeitsgefühl in Ost und West suggerieren sollen. Damit hängt ein revanchistisches Moment eng zusammen, das am ehesten mit der Formel des 'Deutschland, Deutschland über alles' charakterisiert ist», in: Hexelschneider/John, ibid., p. 13. Pour davantage de détails sur cette question, cf. Georg Herde, «Regierung Kohl und die Revanchistenverbände», Marxistische Blätter, 6 (1983), p. 97-102.

32 «nationalistische Überhebung» et "romantische Deutschtümelei», in: Alfred KosıNG, Nation in Geschichte und Gegenwart, Berlin, Dietz Verlag, 1976, p. 277.

33 «Eine kulturelle [...] und ideologische Einheit kann es bei antagonistischen Klassen, die in einer Nation zusammenleben, nicht geben, geschweige denn zwischen Nationen unterschiedlicher Gesellschaftsordnung. Die Kultur trägt in Klassengesellschaften immer Klassencharakter», in: HexeLSCHNEIDER/JOHN, Kultur als einigendes Band? (note 27), p. 43. 
de Kulturnation est devenu problématique en RFA aussi mais pour d'autres raisons: la nation risquait de s'épuiser en une «nation culturelle» sans expression politique ${ }^{(34)}$. Le statut de la RFA lui-même devait être redéfini: la RFA ne pouvait plus prétendre représenter la seule incarnation du Reich, et elle ne pouvait pas non plus se présenter comme un État définitif. Cette question a été résolue par le Tribunal de Karlsruhe, qui a admis l'identité de la République fédérale au Reich dont elle est l'incarnation, mais en précisant que les deux États sont des parties d'un «État allemand» qui continue d'exister ${ }^{(35)}$. Ce jugement a été à l'origine de la thèse de «l'identité partielle», Teilidentität, des deux États avec l'Allemagne.

La chute du Mur de Berlin puis les négociations préalables à la signature du traité d'unification obligent à définir une forme d'action publique sur la culture radicalement nouvelle pour la RFA. Pour la première fois, on admet que l'intervention de la Fédération (le Bund) dans le domaine de la vie culturelle intérieure va être nécessaire, car il était clair que les institutions culturelles est-allemandes ne pouvaient pas assurer elles-mêmes du jour au lendemain leur survie sur le plan financier. Mais en même temps, il n'était pas question pour la Fédération de définir des modes d'action dans le domaine culturel qui auraient eu vocation à se pérenniser, il s'agissait uniquement de financer un sursis, de proposer une aide transitoire pour éviter que le passage entre une politique culturelle dictatoriale, centralisée, où tout ou presque était pris en charge par l'État, à une politique culturelle démocratique ne se traduise par un effondrement de toutes les institutions culturelles est-allemandes. La situation était là encore un peu particulière à Berlin, dans la mesure où la division pendant 40 ans avait eu tendance à créer une densité de théâtres, de maisons de la culture, de cinémas, de bibliothèques ${ }^{(36)}$, etc., plus importante que dans une ville «normale». Tout existait en quelque sorte en double. Il fallait donc à la fois sauver ces institutions culturelles et faire en sorte qu'elles puissent acquérir une autonomie financière permettant à la Fédération de se retirer dans un second temps. Cela a fait l'objet de négociations dans le cadre des discussions autour de la rédaction du traité d'unification entré en vigueur le 3 octobre 1990. La phrase sur laquelle les deux États se sont mis d'accord est la suivante: «La substance culturelle [dans les nouveaux Länder] ne doit pas connaître de dommages» ${ }^{(37)}$. Nous proposons donc

34 Cf. sur cette question les analyses de Karl Jaspers: Karl JAspers, Freiheit und Wiedervereinigung. Aufgaben deutscher Politik, Munich, Piper, 1960, ainsi que Karl Jaspers, Wohin treibt die Bundesrepublik? Tatsachen, Gefahren, Chancen, Munich, Piper, 1966. Il montre que la RFA n'est pas identique au Reich, mais est une construction nouvelle qu'il est urgent de définir, et aborde en particulier la question de l'abandon de l'impératif d'unification. On pourrait également se reporter à la position de Gustav Heinemann.

35 Cf. «Beschluß des Bundesverfassungsgerichts vom 7. Juli 1975 über Verfassungsbeschwerden gegen die Verträge von Moskau und Warschau», in: Rechtsstellung Deutschlands: Völkerrechtliche Verträge und andere rechtsgeltende Akte, Munich, DTV, 1985.

36 Cf. Elisa Goudin, Culture et action publique en Allemagne. L'impact de l'unification, Paris, Éditions Connaissances et Savoirs, 2004.

37 Cf. Artikel 35: «(1) In den Jahren der Teilung waren Kunst und Kultur - trotz unterschiedlicher Entwicklung der beiden Staaten in Deutschland - eine Grundlage der fortbestehenden Einheit der deutschen Nation. Sie leisten im Prozeß der staatlichen Einheit der Deutschen auf dem Weg zur europäischen Einigung einen eigenständigen und unverzichtbaren Beitrag. Stellung und Ansehen 
de montrer de quelles façons la Fédération a tenté, dans le contexte de l'Allemagne réunifiée entre 1990 et 1994, de remplir cette exigence, quels programmes ont été mis en œuvre et sur quelles convictions ils reposent.

\section{Les programmes d'action mis en place après 1990 par le nouvel État réunifié}

Les dispositions en matière de politique culturelle suite à l'unification se déclinent en plusieurs programmes d'action qui ont concerné l'ex-RDA tout entière, et tout particulièrement Berlin et la Saxe en raison de la densité particulièrement importante d'institutions culturelles qui les caractérisait. Ces mesures n'ont pas toujours permis d'éviter les fermetures. 60 \% des chômeurs dans le secteur culturel à la fin des années 1990 vivaient en Saxe ou à Berlin-Est ${ }^{(38)}$, mais elles ont tenté de limiter les conséquences du passage brutal à l'économie de marché.

\section{A. Le programme de maintien de la substance culturelle, "Substanzerhaltungsprogramm»}

Ce programme a été mis en place par le ministère de l'Intérieur en coopération avec un conseil spécialisé (Fachbeirat) constitué de spécialistes des questions culturelles. Dès son adoption, en février 1991, 10 millions de DM ont été attribués à Berlin, comme à chaque nouveau Land, afin d'apporter une aide d'urgence aux institutions culturelles qui se trouvaient dans une impasse financière. Il est conçu pour être une aide transitoire: à terme, les Länder de l'Est devaient être en mesure de financer euxmêmes leurs activités culturelles, mais ce fut loin d'être le cas. Cette notion d'aide transitoire, de sursis financé par la Fédération, distingue ce programme d'un classique programme de subvention de la culture. Il s'agissait d'éviter un effondrement économique immédiat, et non de mettre en place des structures d'aides qui auraient eu vocation à se pérenniser. C'est la raison pour laquelle dans ce programme, les institutions concernées devaient financer elles-mêmes au moins $50 \%$ de leurs activités. La seule exception à cette règle générale est la ville de Berlin, où la Fédération a décidé de financer la restructuration culturelle pour les trois quarts, car Berlin possédait une très forte concentration d'institutions culturelles que la ville ne pourrait en aucun cas financer elle-même pour moitié, en raison du rôle de vitrine qu'elle avait joué pendant la division. Ce programme de maintien de la substance culturelle avait pour priorités: - les institutions ou initiatives de rang européen ou international,

- les théâtres, musées, orchestres, bibliothèques, qui sont prédominants pour leur région,

- les manifestations mises en place dans le passé, reprises régulièrement, et significatives,

eines vereinten Deutschlands in der Welt hängen außer von seinem politischen Gewicht und seiner wirtschaftlichen Leistungskraft ebenso von seiner Bedeutung als Kulturstaat ab. Vorrangiges Ziel der Auswärtigen Kulturpolitik ist der Kulturaustausch auf der Grundlage partnerschaftlicher Zusammenarbeit.

(2) Die kulturelle Substanz in dem in Artikel 3 genannten Gebiet darf keinen Schaden nehmen. »

38 D’après Anja Scholz et Cornelia Waldkircher-Heyne, Entwicklungstrends von Kunst, Kultur und Medien in den neuen Bundesländern, Schriftenreihe des ifo Instituts für Wirtschaftsforschung, Berlin/Munich, Duncker \& Humblot, 1994. 
- la promotion culturelle du film,

- les lieux commémoratifs ${ }^{(39)}$.

Concrètement, la ville de Berlin, comme chaque nouveau Land, a été chargée au début de l'année 1991 d'établir une liste des institutions culturelles qui pouvaient entrer dans le cadre de ce programme, «Förderliste». Ensuite, la Fédération ${ }^{(40)}$ a décidé si oui ou non telle ou telle institution culturelle donnée pouvait bien figurer sur la liste, mais la mise en œuvre finale de ce programme d'action a été le fait des Länder: chaque Land a réparti la somme d'argent globale versée par la Fédération entre les institutions culturelles.

Au total, la Fédération a dépensé 1,5 milliard de DM dans le cadre de ce programme ${ }^{(41)}$. L'aide s'est étendue sur trois ans, de façon légèrement dégressive: en 1991,

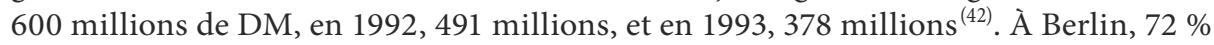
de cette aide ont été attribués aux théâtres. Ce chiffre, qui peut paraître démesuré, s'explique par plusieurs raisons: tout d'abord les théâtres coûtent cher comparativement à d'autres institutions culturelles, notamment en personnel. Le retrait du SED de ses responsabilités à l'égard des théâtres est-allemands a donc créé un important vide à combler. De plus, la Fédération a toujours souhaité privilégier une forme «vivante» d'expression artistique. Par ailleurs, une priorité très claire est accordée, dans la définition des critères à remplir pour pouvoir prétendre à cette aide, aux institutions culturelles ayant une résonance extrarégionale, voire européenne ${ }^{(43)}$. Ceci ne doit pas nous surprendre, car il s'agit de la vocation de la Fédération, à la différence de la mission des communes et des Länder qui concerne les activités culturelles locales.

\section{B. Le programme portant sur l'infrastructure culturelle, "Infrastukturprogramm »}

Ce programme repose juridiquement sur l'article 91a de la Loi fondamentale, en vertu duquel la Fédération est compétente pour établir des conditions de vie comparables dans les différentes régions allemandes, et donc pour atténuer les inégalités régionales. Or, la densité des infrastructures culturelles est un élément de la définition du cadre de vie. Ce programme a été mis en œuvre entre 1991 et 1993, avec la dotation budgétaire suivante: 300 millions de DM en 1991, 246 millions en 1992, et 184 millions en 1993, soit un total de 730 millions ${ }^{(44)}$. De même que pour le programme précédent, la

39 Les termes exacts utilisés dans le programme sont les suivants:

- Einrichtungen bzw. Vorhaben von europäischem/nationalem Rang

- Theater, Museen, Orchester, Bibliotheken, die überwiegend für ihre Region von Bedeutung sind

- in der Vergangenheit begonnene, wiederkehrende bedeutsame Veranstaltungen

- kulturelle Filmförderung

- Mahn- und Gedenkstätten.

40 La Fédération a chargé un conseil spécialisé (Fachgremium) de statuer sur ces questions de financement.

41 Ce chiffre est donné dans l'ouvrage de synthèse publié par le ministère fédéral de l'Intérieur sur les cinq premières années de l'aide financière à la culture est-allemande: Bundesministerium des Innern, 5 Jahre Kulturförderung für die neuen Länder, Bonn, Pfälzische Verlagsanstalt GmbH, 1996.

42 Ce programme est parfois nommé «Übergangsfinanzierung zur Erhaltung der kulturellen Substanz im Gebiet der ehemaligen DDR», ou encore «Sonderfonds Förderung gefährdeter kultureller Einrichtungen und Veranstaltungen insbesondere von europäischem Rang».

43 On trouve l'expression «Förderung kultureller Einrichtungen und Veranstaltungen insbesondere von europäischem Rang», Kabinettvorlage des BMI, 5 novembre 1990.

44 Cf. Bundesministerium des Innern, 5 Jahre Kulturförderung (note 41). 
Fédération a exigé que $50 \%$ des coûts soient pris en charge par l'institution culturelle concernée. Ce principe se situe dans la lignée des efforts de la Fédération pour obliger les institutions culturelles est-allemandes à rompre avec la logique de l'assistance étatique. D’autre part, ce programme est une réponse à la préoccupation exprimée par la commission culturelle qui avait siégé avant l'unification et avait souligné la nécessité d'apporter une aide au maintien de l'infrastructure culturelle:

«Pour parvenir à une compensation des effets de la division de l'Allemagne, il faut renforcer en priorité le maintien et le développement de l'infrastructure culturelle, ainsi que les initiatives culturelles sur le territoire de l'ancienne RDA» ${ }^{(45)}$.

Plusieurs secteurs d'action ont été définis dans le cadre de ce programme:

«le théâtre, les arts plastiques, la musique, la littérature, le film et les médias, les bibliothèques, les musées, les archives et collections, l'entretien des monuments, la formation pour jeunes et pour adultes, la socio-culture, la culture de la patrie, l'art populaire et la civilisation historique ${ }^{(46)}$.

L'objectif était explicitement de mettre en place une sorte de contrepoids au programme de maintien de la substance culturelle, car cette aide financière s'adressait surtout aux petites institutions culturelles. Il s'agissait donc, par le biais de subventions au secteur culturel, de corriger les déséquilibres régionaux qui existaient entre les anciens et les nouveaux Länder, mais aussi entre les nouveaux Länder eux-mêmes. Ce programme a donc plutôt défavorisé Berlin. Au total, la répartition s'est faite de la façon suivante: Berlin: $5 \%$, Brandebourg: $21 \%$, Mecklenbourg-Poméranie occidentale: $17 \%$, Saxe: $23 \%$, Saxe-Anhalt: $18 \%$, Thuringe : $16 \%$.

Par ailleurs, ce programme devait apporter une réponse au problème posé par la disparition de nombreuses entreprises de RDA. Les entreprises populaires de l'exRDA comme lieux de culture comptaient parmi les bénéficiaires de ce programme. Le partage des institutions concernées s'est effectué là encore selon le critère de la capacité à payer $51 \%$ des coûts de la restructuration.

\section{Le programme d'atténuation des conséquences de la division}

Le programme "Milderung von Teilungsfolgen» est un autre programme fédéral mis en place par le ministère de l'Intérieur. Le budget qui l'accompagnait a été très fortement dégressif, puis très légèrement en hausse: 18 millions en 1991, puis seulement 3 millions en 1992 et 4 en 1993. De même que pour les autres programmes, il était transitoire, n'a duré que trois ans et exigeait des institutions culturelles concernées qu'elles apportent un volume financier supplémentaire correspondant à $51 \%$ de l'aide totale à la restructuration.

L'originalité de ce programme tient au fait qu'il a été conçu explicitement pour accompagner l'unification en lui ajoutant un volet culturel:

45 «Zum Ausgleich der Auswirkungen der Teilung Deutschlands sind die Erhaltung und der Ausbau der kulturellen Infrastruktur sowie kulturelle Maßnahmen in den Gebieten der ehemaligen DDR bevorzugt zu stärken», in: Manfred Ackermann, Der kulturelle Einigungsprozeß. Schwerpunkt: Substanzerhaltung, Forum Deutsche Einheit, Perspektiven und Argumente, n 7 (1991), p. 37.

46 «darstellende Kunst, bildende Kunst, Musik, Literatur, Film und Medien, Bibliotheken und Büchereien, Museen, Archive und Sammlungen, Denkmalpflege, Jugend- und Erwachsenenbildung, Soziokultur, Heimatpflege, Volkskunst und historische Landeskunde». 
«L'objectif est de démonter les handicaps qui pèsent sur les manifestations culturelles dans les Länder de l'Est, de relier entre eux des secteurs culturels et des organisations dans l'Est et dans l'Ouest de l'Allemagne [...] et de rendre possibles des projets interallemands sur le long terme, ou le cas échéant de les reconduire » ${ }^{(47)}$.

Cette volonté de favoriser une étroite coopération interallemande concerne tout particulièrement Berlin. Ce programme devait apporter les moyens financiers nécessaires pour inciter les artistes de RFA et de l'ex-RDA à travailler ensemble, notamment à Berlin, afin d'œuvrer à réunifier la ville sur le plan culturel. L'enjeu est donc, au-delà de la mise en place d'une coopération culturelle plus étroite, de favoriser la volonté des Allemands de réaliser ensemble un projet de société partagé. En ce sens, ce programme repose sur une conception d'une nation allemande en reconstruction. Alain Renaut définit l'approche rationaliste de la nation, par opposition à l'approche organiciste, comme suit: «La nationalité est en effet pensée en termes, non pas d'adhésion pure et simple, ni d'appartenance pure et simple, mais d'éducabilité » ${ }^{(48)}$. C'est bien autour de cette notion d'éducabilité que se noue le lien entre ce programme fédéral et la création d'une nouvelle identité allemande née de l'unification. Il ne s'agit pas de créer une culture allemande parfaitement homogène, une communauté de culture, mais bien de faire en sorte que les Allemands, aussi bien de l'Est que de l'Ouest, soient davantage en mesure de réapprendre à se connaître après les années de séparation.

\section{Le programme de qualification dans le domaine culturel, "Qualifizierungsprogramm»}

Ce programme fédéral a été mis en place en 1992 par le ministère fédéral de la Science, de la Formation, de la Recherche et de la Technologie. Il a été doté au total, sur les trois ans d'action, d'un budget de 7 millions de $\mathrm{DM}^{(49)}$. Sa particularité est d'apporter une réponse au besoin d'information, de qualification et de conseils des employés des institutions culturelles est-allemandes. Il a notamment permis la mise en place de l'IBF-K, «Service d'information, de conseil et de formation continue pour les administrations culturelles est-allemandes » ${ }^{(50)}$. Cet organisme est composé essentiellement d'institutions culturelles originaires de l'Ouest, qui s'occupent de la vie culturelle locale dans les nouveaux Länder, et donc en particulier dans l'exBerlin-Est ${ }^{(51)}$. Il s'agit de mettre en place un réseau de structures non-étatiques capables de proposer aux institutions culturelles est-allemandes des prestations de conseil et de formation. L'objectif est, à terme, d'assurer la décentralisation culturelle

47 «Ziel ist es, Benachteiligungen bei kulturellen Veranstaltungen in den Ost-Ländern abzubauen, Kulturbereiche und Organisationen in Ost- und Westdeutschland organisatorisch miteinander zu vernetzen [...] und langfristige gesamtdeutsche Projekte zu ermöglichen bzw. weiterzuführen», in: Scholz/WALdKircher-Heyne, Entwicklungstrends von Kunst, Kultur und Medien (note 38), p. 167.

48 Alain Renaut, Présentation de l'ouvrage de Johann Gottlieb Fichte, Discours à la nation allemande (1806), Paris, Imprimerie nationale, 1992, p. 41.

49 Cf. Presse- und Informationsamt der Bundesregierung, So fördert der Bund Kunst und Kultur. Innerstaatliche Kulturförderung und Auswärtige Kulturpolitik, 1996.

50 «Informations-, Beratungs-, und Fortbildungsdienst für ostdeutsche Kulturverwaltungen».

51 Parmi ces institutions, on trouve le Deutscher Städtetag, le Deutscher Landkreistag, le Deutscher Städte- und Gemeindebund. Et l'une des institutions les plus importantes de ce IBF-K est la Stiftung für kulturelle Weiterbildung und Kulturberatung in Ost-Berlin. 
de l'ex-RDA. Ce programme a permis la création du premier embryon d'économie de la culture dans les nouveaux Länder, en aidant financièrement les galeries, les librairies, les maisons d'édition, les bureaux de design et d'architecture. Le souci constant des concepteurs de ce programme d'action était de s'orienter vers les questions pratiques: aides aux nouvelles entreprises du secteur culturel, conseils, soutien des initiatives culturelles ayant une vocation commerciale, etc., afin de favoriser concrètement la transition des structures de gestion culturelle est-allemandes vers l'économie de marché.

\section{E. Les autres formes d'intervention fédérale}

La Fédération a également subventionné un certain nombre d'actions ponctuelles, comme la fondation du Fonds culturel, "Stiftung Kulturfonds», qui correspond à l'ancien Fonds culturel de RDA, «Kulturfonds DDR», et dont la vocation est d'aider les artistes est-allemands en difficulté. Au total, le financement de cette fondation sur quatre ans s'est élevé à 20,5 millions de $\mathrm{DM}^{(52)}$, soit environ cinq millions de DM par an. La Fédération s'est engagée par contrat à assurer la survie financière de cette institution seulement jusqu'au 31 décembre 1994 (paragraphe 35.6 du traité d'unification). Cette institution se trouvait au moment de l'unification dans une véritable impasse financière, mais elle avait un rôle social déterminant, ce qui explique qu'elle ait fait partie des priorités de la Fédération.

Quant au programme "Essor de l'Est», "Aufschwung Ost», il a été mis en place en 1991 et a été doté d'un budget global de 230 millions de $\mathrm{DM}^{(53)}$. Il comportait quelques dispositions sur l'aide au développement de l'infrastructure culturelle est-allemande, mais de façon relativement marginale, puisque ce programme était avant tout un programme économique, dont la vocation était d'accélérer le développement économique des nouveaux Länder.

Ensuite, le programme intitulé "Phares", le "Leuchtturm-Programm», a permis d'apporter une aide ponctuelle pour entretenir des bâtiments, ou moderniser certaines constructions qui servaient de lieux de création culturelle mais menaçaient de s'effondrer. Ici, la Fédération a rempli une mission que des entreprises traditionnelles ne pouvaient pas prendre à leur charge. Au total, la Fédération a ainsi dépensé 20 millions ${ }^{(54)}$ de DM par an, afin de régler le problème du manque chronique d'investissements.

Il faudrait également mentionner la parution en 1996 d’un ouvrage intitulé «Abécédaire de l'artiste», Künstlerfibel, établi par le ministère de l'Économie en coopération avec le Conseil culturel, Kulturrat, afin d'aider les artistes est-allemands en difficulté à connaître les différentes aides auxquelles ils ont droit, les organismes de conseils auxquels ils peuvent s'adresser, etc.

52 Ce chiffre est celui qui est indiqué dans l'ouvrage de Klaus von BEYME, Kulturpolitik und nationale Identität. Studien zur Kulturpolitik zwischen staatlicher Steuerung und gesellschaftlicher Autonomie, Wiesbaden, Westdeutscher Verlag, 1998. D'autres sources proposent des chiffres très proches, variant toujours entre 19 et 21 millions de DM.

53 Cf. Bundesminister des Inneren, Bericht zum Stand der Übergangsfinanzierung der Kultur im Beitrittsgebiet, Bonn, 20 septembre 1991, p. 2.

54 D’après le chiffre indiqué par le ministère fédéral de l’Intérieur, in : ibid., p. 2. 
Pour conclure, ajoutons que certains souhaitaient, lors de la rédaction du traité d'unification, que la protection de la culture entre dans la liste des prérogatives de l'État, au même titre que la protection de l'environnement par exemple. Cela n'a pas été le cas mais la Fédération a pu, grâce à l'article 35 de ce traité, poser les principes d'une action de grande envergure dans le domaine de la restructuration des institutions culturelles est-allemandes. Elle a été amenée à offrir un sursis aux institutions culturelles est-allemandes, afin de leur laisser du temps pour s'adapter aux mécanismes du marché. Certains y ont même vu un danger dans la mesure où cela remettait en cause le principe de l'autonomie des Länder dans le domaine culturel (Kulturhoheit der Länder) et constituerait donc une entorse à la Loi fondamentale. L'idée d'un empiètement sur le terrain des Länder est souvent définie comme un danger, une menace ${ }^{(55)}$ par les partisans d'une interprétation stricte du fédéralisme culturel. Ainsi lors de l'unification, la fusion des fonds de Berlin-Est et de ceux de Berlin-Ouest au sein de la Fondation du patrimoine prussien, Stiftung Preußischer Kulturbesitz ${ }^{(56)}$, a été dénoncée, dans la mesure où il était décidé que cette institution unifiée serait financée à hauteur de $75 \%$ par la Fédération.

Toutefois il serait erroné de considérer que la politique culturelle de l'Allemagne unifiée est devenue une politique centralisée, définie depuis Berlin, comme l'était celle de la RDA. Les programmes fédéraux n'étaient pas conçus comme un soutien pérenne, ils devaient seulement «acheter du temps» pour sauver la culture ${ }^{(57)}$. L'autre critique récurrente porte sur le supposé manque de responsabilité des acteurs culturels estallemands. Une enquête effectuée par Eberhard Diepgen, membre de la CDU, qui était alors maire de Berlin, conclut en 2000 à la nécessité de "dépolitiser les entreprises culturelles implantées à Berlin ${ }^{(58)}$. Par l'expression de "dépolitiser», il faut entendre dans ce cas précis une volonté de supprimer l'influence des autorités publiques. Selon lui, «la prise directe du législatif et de l'exécutif sur les budgets, la programmation et les décisions concernant le personnel a renforcé l'attitude passive de nombreuses institutions culturelles face au problème des coûts. C'est pourquoi les "entreprises culturelles d'État" devraient être transformées en entreprises libres » ${ }^{(59)}$. La mainmise de l'État sur les institutions culturelles conduirait donc, dans cette perspective, à déresponsabiliser les acteurs culturels eux-mêmes. Ces propos ont bien sûr suscité de

55 Une des conséquences de cela est le fait que la Fédération n'est souvent perçue que comme une instance de paiement, mais qui n'est pas habilitée à définir des directives, à poser des principes d'action. Ainsi par exemple, la fondation du patrimoine prussien, Stiftung preußischer Kulturbesitz, est financée à hauteur de $20 \%$ par les Länder, mais ces derniers détiennent $40 \%$ des voix au conseil de gestion, cette disproportion nous semble se situer dans la lignée des efforts de certains représentants des Länder pour éloigner la Fédération le plus possible des décisions de politique culturelle.

56 Cette institution avait été fondée le 25 juillet 1957.

57 Le dernier ministre de la Culture de RDA, Herbert Schirmer, a utilisé l'expression «tortillard dans le train express de l'unification », «Bummelzug im Einigungsexpress», à propos du secteur culturel.

58 «Eine Entpolitisierung der Berliner Kulturbetriebe», in: «Berliner Kulturlandschaft soll reformiert werden - 'Entpolitisierung' der Theater gefordert», Neue Musikzeitung, 04.04.2000.

59 «Der direkte Zugriff von Legislative und Exekutive auf Budgets, Programme und Personalentscheidungen habe die passive Haltung vieler Kulturinstitutionen gegenüber dem Kostenproblem verfestigt. Deshalb sollten 'kulturelle Staatsbetriebe' in freie Unternehmensformen überführt werden ", Neue Musikzeitung, ibid. 
vives réactions, notamment de l'intendant du Berliner Ensemble, Claus Peymann, qui reproche à Eberhard Diepgen de conduire une argumentation simpliste: «La ville de Berlin montre à nouveau la figure grotesque d'un individu terre à terre » ${ }^{(60)}$.

Indépendamment des critiques formulées contre les programmes fédéraux, il est indéniable que les nouveaux Länder, et notamment Berlin-Est, se sont trouvés dans une situation nettement plus confortable que les États d'Europe centrale et orientale par exemple, qui ont été contraints de financer eux-mêmes en grande partie leur propre changement sociétal, notamment dans le domaine de la culture. Toutefois cela n’a pas empêché la fermeture de très nombreux établissements culturels de Berlin-Est. Et, d'une façon générale, le sentiment des acteurs culturels est-allemands a souvent été celui d'une mise à l'écart. Beaucoup étaient convaincus que la RDA avait beaucoup à apporter, ils se fondaient sur des éléments aussi disparates que les mouvements pour la démocratie, les liens culturels privilégiés avec l'Europe centrale, la présence de la culture dans la vie professionnelle, la démocratisation de l'accès à la pratique culturelle dans le cadre des collectifs artistiques dans les entreprises, etc. Or, à l'image de la RDA elle-même, rien de tout cela n'a été importé dans la nouvelle Allemagne, ce qui a créé des ressentiments importants. C'est le sens de l'assertion de Günter Grass :

«Des sommes considérables ont été injectées à l’Est. Avec quelques résultats, il va sans dire. Mais la culture, ce n'est pas uniquement la restauration à coup de millions du patrimoine architectural. L’erreur la plus grossière a été de décréter que la culture de l'ancienne RDA, à l'instar de ses industries déclarées inadaptées et dépassées, était bonne à être envoyée à la casse. D’où une immense amertume, notamment chez les plus âgés » ${ }^{(61)}$.

Jürgen Habermas insiste quant à lui sur le danger que représentait pour le maintien du consensus social cette gestion à court terme, qui visait une autonomie financière rapide des institutions culturelles:

«La liquidation administrative des académies, des universités, des musées, la réorganisation des théâtres, du film et de la littérature sur le modèle bien éprouvé à l'Ouest du marché et des subventions, ont des effets bien plus graves que la destruction des capacités de production dans d'autres secteurs ${ }^{(62)}$.

$\mathrm{Au}$ total, la politique culturelle de la RFA a donc été nettement plus politisée après 1990, elle a fait l'objet de débats nettement plus virulents que ce qui caractérisait la politique culturelle de la RFA d'avant 1990. Ces débats portaient notamment sur la façon dont il fallait intervenir pour sauvegarder la «substance culturelle est-allemande » et gérer l'héritage culturel de la RDA. Cela s'illustre dans la gestion du musée historique de Berlin. Le premier gouvernement démocratiquement élu en RDA a fait fermer ce musée, qui présentait l'histoire de l'Allemagne uniquement sous l'angle d'une succession de luttes des classes. Et il a ensuite été rouvert, avec un objectif radicalement

60 «Die Stadt zeigt wieder ihre Banausenfratze», Claus Peymann sur la radio berlinoise «radio kultur», cité dans l'article de la Neue Musikzeitung, ibid.

61 Interview de Günter Grass, Le Figaro, 12 octobre 1995.

62 Jürgen Habermas, «Was bedeutet 'Aufarbeitung der Vergangenheit' heute? Bemerkungen zur 'doppelten Vergangenheit'»(1990), in: Die Moderne, ein unvollendetes Projekt. Philosophisch-politische Aufsätze, 1977-1992, Leipzig, Reclam, 1992, p. 264, cité par Pascale LABORIER, in : Culture et édification nationale en Allemagne. Genèse des politiques de la culture, doctorat sous la direction de M. le professeur Jean Leca, Institut d'études politiques de Paris, 1996, non publié, p. 697. 
différent, celui de contribuer à long terme à rapprocher les deux Allemagnes, et de permettre une compréhension plus grande du passé commun des deux anciens États allemands. Ce musée emploie aujourd'hui un tiers d'employés originaires de RFA et deux tiers d'employés de l'ex-RDA ${ }^{(63)}$ et se nomme l'Arsenal.

Notons enfin que la situation juridique floue dans les nouveaux Länder après l'unification a permis à de nombreux projets culturels alternatifs de voir le jour sans que cela ne soit lié à la politique culturelle. Des projets de squats d'artistes comme la Kulturbrauerei ou le Tacheles, en marge des mesures de politique culturelle, ont fait la réputation de Berlin dans les années 1990. Il s'agissait d’utiliser les lieux abandonnés, en développant parfois une véritable métonymie entre le lieu et l'état d'esprit des artistes. La devise du Tacheles était par exemple: "Les idéaux sont ruinés, sauvez la ruine» ${ }^{(64)}$. À cet égard, même si d'autres lieux similaires ont vu le jour dans d'autres villes de l'ex-RDA, Berlin fait figure de référence, en raison de sa situation géographique très particulière d'ex-ville du Mur. L'emplacement occupé pendant 28 ans par le Mur ainsi que toutes les anciennes usines fermées constituaient autant d'opportunités spatiales à investir pour les artistes du monde entier qui ont convergé à Berlin. De la même façon, lorsqu'il a été décidé de mettre en place en 1997 une grande exposition temporaire nommée Deutschlandbilder, qui présentait ensemble des œuvres d'artistes est-allemands et ouest-allemands, le choix du lieu s'est porté sur le bâtiment Gropius, situé sur l'emplacement de ce qui était Berlin-Ouest pendant la division de l'Allemagne, mais juste au niveau de la frontière avec Berlin-Est, tout près des lieux commémoratifs de la fin du régime national-socialiste, et en particulier de l'exposition «Topologie de la Terreur», "Topologie des Terrors", un emplacement qui était donc chargé de très nombreux symboles propres à Berlin.

\section{Résumé}

Cet article retrace les grandes lignes de la politique culturelle de la RDA. La politique culturelle de Berlin-Est est analysée dans son rôle de propagation de l'orthodoxie politique, mais aussi comme une pratique sociale, en montrant son rôle dans les entreprises par exemple. Dans un second temps nous examinons les mesures de transition mises en place au moment de la réunification afin de redéfinir des modalités d'action de l'État sur la culture qui puissent éviter que ne se perde la "substance culturelle est-allemande", conformément à l'article 35 du traité d'unification. L'idée qui sous-tend ces différents programmes fédéraux est qu'il fallait laisser du temps aux institutions culturelles pour s'adapter, ils ne prétendaient pas représenter une solution de long terme, mais seulement "acheter du temps" pour la culture. Nous montrons les logiques sur lesquels ils reposent.

63 Pour une étude détaillée de ce revirement, cf. par exemple l'étude de Christoph StöLzL, «Un musée fait une pirouette - Le musée historique allemand et l'Arsenal - Un laboratoire de l'unification", in: Hermann Glaser, Ce qui reste, ce qui sera. Le changement culturel dans les nouveaux Länder, Bonn, Internationes, 1995, p. 161-164.

64 «Die Ideale sind ruiniert, rettet die Ruine.» 


\section{Zusammenfassung}

Dieser Artikel beschreibt zunächst die Kulturpolitik der DDR: sowohl deren Funktion zur Beförderung von SED-Botschaften als auch deren soziale Praxis in den Unternehmen. Anschließend werden die Übergangsmaßnahmen analysiert, die zum Zeitpunkt der Vereinigung definiert wurden und die verhindern sollten, dass die „ostdeutsche kulturelle Substanz"verloren gehe (gemäß Artikel 35 des Einigungsvertrages). Den verschiedenen Programmen des Bundes lag die Idee zugrunde, dass man den kulturellen Institutionen Zeit lassen müsse, sich anzupassen. Der Bund sollte in dieser Übergangsphase ,Zeit' für die Kultur, kaufen'. 